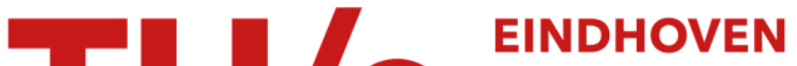 \\ UNIVERSITY OF \\ TECHNOLOGY
}

\section{Parametrizing hydrogen bond interactions in dissipative particle dynamics simulations}

Citation for published version (APA):

Kacar, G., \& de With, G. (2020). Parametrizing hydrogen bond interactions in dissipative particle dynamics simulations: the case of water, methanol and their binary mixtures. Journal of Molecular Liquids, 302, [112581]. https://doi.org/10.1016/j.molliq.2020.112581

\section{Document license:}

TAVERNE

DOI:

10.1016/j.molliq.2020.112581

Document status and date:

Published: 15/03/2020

\section{Document Version:}

Publisher's PDF, also known as Version of Record (includes final page, issue and volume numbers)

\section{Please check the document version of this publication:}

- A submitted manuscript is the version of the article upon submission and before peer-review. There can be important differences between the submitted version and the official published version of record. People interested in the research are advised to contact the author for the final version of the publication, or visit the $\mathrm{DOI}$ to the publisher's website.

- The final author version and the galley proof are versions of the publication after peer review.

- The final published version features the final layout of the paper including the volume, issue and page numbers.

Link to publication

\section{General rights}

Copyright and moral rights for the publications made accessible in the public portal are retained by the authors and/or other copyright owners and it is a condition of accessing publications that users recognise and abide by the legal requirements associated with these rights.

- Users may download and print one copy of any publication from the public portal for the purpose of private study or research.

- You may not further distribute the material or use it for any profit-making activity or commercial gain

- You may freely distribute the URL identifying the publication in the public portal.

If the publication is distributed under the terms of Article 25fa of the Dutch Copyright Act, indicated by the "Taverne" license above, please follow below link for the End User Agreement:

www.tue.nl/taverne

Take down policy

If you believe that this document breaches copyright please contact us at:

openaccess@tue.nl

providing details and we will investigate your claim. 


\title{
Parametrizing hydrogen bond interactions in dissipative particle dynamics simulations: The case of water, methanol and their binary mixtures
}

\author{
Gokhan Kacar ${ }^{\mathrm{a}, *}$, Gijsbertus de With ${ }^{\mathrm{b}, *}$ \\ a Department of Genetics and Bioengineering, Faculty of Engineering, Trakya University, 22030 Edirne, Turkey \\ ${ }^{\mathrm{b}}$ Laboratory of Physical Chemistry, Faculty of Chemical Engineering and Chemistry, Eindhoven University of Technology, Eindhoven, the Netherlands
}

\section{A R T I C L E I N F O}

\section{Article history:}

Received 5 November 2019

Received in revised form 21 January 2020

Accepted 26 January 2020

Available online 27 January 2020

\section{Keywords:}

Water

Methanol

Water-methanol mixtures

Coarse-grained simulations

Dissipative particle dynamics

\begin{abstract}
A B S T R A C T
Simulating water has always been a challenge. Due to the intrinsic hydrogen bond interactions, water exhibits structural properties, such as a tetrahedral coordination resulting in a specific Radial Distribution Function (RDF), which are not trivial to predict computationally. In this paper, we attempt to use coarse-grained Dissipative Particle Dynamics (DPD) simulations to parameterize the hydrogen bond interactions without violating the classical DPD framework. We model the hydrogen bond interactions by incorporating a Morse potential, where the parameters are computed by taking the experimental enthalpy of evaporation and hydrogen bond distances as reference. We show that with the proposed procedure the RDF, the coordination number, the isothermal compressibility, and the three-body angular distributions (to demonstrate the tetrahedral structure) of pure water are predicted in great extent compatible with the experiments. To test the applicability of the procedure to mixtures, we simulated pure methanol and methanol/water mixtures at different molar fractions. The predicted RDF profiles for methanol-methanol, methanol-water and water-water represent the characteristic experimental RDF behavior. Moreover, the calculated negative excess volumes as a function of mole fraction compare quite well with the experimentally observed excess volumes. Our findings motivate the further development and use of DPD simulations in modeling hydrogen bond interactions, which are crucial not only in water (or alcohols), but in more complex systems such as biomolecules, proteins or biopolymers.
\end{abstract}

(c) 2020 Elsevier B.V. All rights reserved.

\section{Introduction}

Coarse-grained simulations are useful tools in reaching larger length scales in molecular simulations [1]. While a coarse-graining process takes place, the number of internal degrees of freedom of specific molecules is decreased significantly due to integrating out the atomistic coordinates. Generally pairwise interactions are defined between (one or more) beads representing each a particular molecule, which are often computed using procedures such as iterative Boltzmann inversion (IBI) [2] and the inverse Monte Carlo method [3]. These methods aim to iteratively map the Radial Distribution Functions (RDFs) of the coarse-grained structure to the atomistic one, until obtaining the correct structure. As alternatives to these techniques, force-matching (FM) and multi-scale coarse-graining (MS-CG) methods are used, which map the atomistic and coarse-grained level forces yielding the proper potential of mean force (PMF) representing many-body effects [4-6]. Recently, the applicability of these force-matching methods has been improved

\footnotetext{
* Corresponding authors.

E-mail addresses: gokhankacar@trakya.edu.tr (G. Kacar), g.dewith@tue.nl (G. de With)
}

significantly by employing iterative force-matching [7] and including the three-body interactions [8]. Although employing these techniques results in a coarse-grained structure of molecules consistent with their atomistic definitions, a significant number of iterations is needed [9]. Moreover, these methods take atomistic molecular dynamics simulations as reference, which eventually makes these techniques dependent on the selected force-field.

Apart from these coarse-grained simulation techniques, Dissipative Particle Dynamics (DPD) is one of the widely employed techniques in the simulation of complex molecular structures. DPD $[10,11]$ is a computationally efficient off-lattice simulation method initially proposed to replace Lattice-Gas [12] and Lattice-Boltzmann techniques [13] for the simulation of the complex hydrodynamic behavior of liquids and supercritical fluids. Later, the DPD method was mapped to the Flory-Huggins mean-field theory allowing the computation of the simulation parameters from experimental FloryHuggins interaction parameters [14]. The calculation of the FloryHuggins parameters from the individual bead solubilities causes quite a versatile parameterization of the coarse-grained DPD interactions. The DPD method has found an enormous area of application in modeling and simulation of complex soft matter [15] from polymers 
$[16,17]$ to proteins $[18,19]$. Nevertheless, the DPD method has employed certain critical assumptions limiting the applicability of the method, one of which is that beads have comparable sizes. Upon coarse-graining of chemical structures, significant size differences up to a factor 3 might occur, as reported in our earlier work [20]. As a possible solution to this drawback, we proposed an alternative parameterization of DPD where the volume of each bead is dictated by its experimental pure liquid density [21]. Another important aspect that is conventionally used in the DPD method is the nature of the non-bonded potential. This is a soft and purely repulsive potential defining pairwise repulsions between the coarsegrained beads, while cohesion is provided by an overall pressure. Therefore, a relative attraction between beads can only be obtained as a result of a lesser repulsion and vice versa. Obviously, this renders the definition of attractive interactions such as hydrogen bonding troublesome or even impossible.

Hydrogen bonding is important in the structure creation of biomolecules, such as DNA, RNA and proteins, for polymers, such as polyethylene glycol (PEG), and for certain small molecules, in particular, water. Therefore, a proper definition of hydrogen bonding in DPD is necessary if a thorough understanding of such structures is aimed for. Vishnyakov et al. defined the hydrogen bond interaction within DPD by incorporating a Morse potential term in addition to the purely repulsive conservative potential [19]. In that paper, the authors studied the helicity of proteins via DPD simulations. Moreover, a Morse term is used in DPD simulations to model proton dissociation [22], proton transport [23] and metal complexation [24]. We later extended their approach by proposing a realistic parameterization scheme, where the Morse potential parameters are computed from atomistic molecular dynamics simulations [25]. By this method, the experimental negative excess volumes of low molecular weight alcohol and water mixtures are predicted successfully. This proposed method is observed to correctly predict the negative excess volumes of PEG 400/water mixtures [26], chain helicity of PEG [27], and recently the drug delivery properties and structure of Pluronics [28].

Modeling and simulation of water has always been a challenge not only at the atomistic level [29] but also at the coarse-grained level $[30,31]$ due to the hydrogen bond interactions which leads to density and structural anomalies. Recently, Urbic et al. predicted such anomalies to a great extent by employing a simple Mercedes-Benz model [32] and an analytical statistical mechanics model [33]. Moreover, there are coarse-grained alternatives proposed, namely the polarizable force field [34,35] and virtual site [36] approaches in the Martini model. Polarizable models have also been used within the DPD framework [37,38] with different levels of coarse-graining. These models are good predictors of water properties but require complex implementation algorithms reducing the computational efficiency in case water is to be used in a highly complex environment.

In this article, we extend our efforts to identify the Morse potential parameters of coarse-grained water beads simulated with the DPD method which is straightforward to implement and computationally efficient. To that purpose, an additional Morse potential term representing the pairwise attraction between water beads is parameterized by taking experimental data as reference. The proposed parameterization is observed to reproduce the experimental data for the RDF, isothermal compressibility and three-body angular distributions of pure water. Moreover, the Morse parameterization of pure methanol yields the proper RDF and the isothermal compressibility. In addition, a set of methanol/water mixtures is simulated and the experimental negative excess volumes are used to identify the hydrogen bond energy, and where the structure is observed to result in a good correspondence with the atomistic one as well. Our work differs from the literature by implementing a separate term for the hydrogen bonds of water with a realistic parameterization scheme, which has the advantage that it controls these interactions well and helps to understand their intrinsic nature more thoroughly.

\section{Method and simulation details}

\subsection{Method}

The simulation method in our study is referred as Dissipative Particle Dynamics (DPD) [14]. In the DPD method, the total non-bonded force $\mathbf{F}_{\boldsymbol{i}}$ acting on a particular coarse-grained bead $i$ is a sum of three forces, namely the conservative $\mathbf{F}_{i j}^{\mathrm{C}}$, random $\mathbf{F}_{i j}^{\mathrm{R}}$ and dissipative force $\mathbf{F}_{i j}^{\mathrm{D}}$,

$$
\mathbf{F}_{i}=\mathbf{F}_{i j}^{\mathrm{C}}+\mathbf{F}_{i j}^{\mathrm{R}}+\mathbf{F}_{i j}^{\mathrm{D}}
$$

The conservative force acts along the vector connecting beads $i$ and $j$, characterizes the equilibrium structure. The conservative force can be computed from the non-bonded purely soft DPD potential $V_{i j}^{C}$ using

$$
\begin{aligned}
& \mathbf{F}_{i j}^{\mathrm{C}}=-\frac{d V_{i j}^{\mathrm{C}}}{d r_{i j}} \hat{\mathbf{r}}_{i j} \text { with } \\
& V_{i j}^{\mathrm{C}}=\left\{\begin{array}{cc}
\frac{a_{i j}}{2}\left(1-\frac{r_{i j}}{r_{\mathrm{DPD}}}\right)^{2} & r_{i j}<r_{\mathrm{DPD}} \\
0 & r_{i j} \geq r_{\mathrm{DPD}}
\end{array}\right.
\end{aligned}
$$

and where $a_{\mathrm{ij}}$ is the strength of the maximum repulsive interaction, $\mathbf{r}_{i j}=\mathbf{r}_{i}-\mathbf{r}_{j}, r_{i j}=\left|\mathbf{r}_{i j}\right|$ and $\hat{\mathbf{r}}_{i j}=\mathbf{r}_{i j} / r_{i j}$. Hence the dimension of the nonbonded DPD repulsion parameter $a_{\mathrm{ij}}$ is energy. This is different from the DPD tradition, where $a_{\mathrm{ij}}$ is often reported with dimension force, but in this paper we do not follow the tradition of presenting formulas in dimensionless form. In other words, we do not assume that $r_{\mathrm{ij}}$ is in $r_{\mathrm{DPD}}$ units and therefore $r_{\mathrm{DPD}}$ explicitly appears in the formulas throughout the manuscript.

In order guarantee that in equilibrium a canonical distribution is obtained, the random and dissipative forces are computed as

$$
\mathbf{F}_{i j}^{\mathrm{R}}=\sigma_{i j} \omega^{\mathrm{R}}\left(r_{i j}\right) \theta_{i j} \hat{\mathbf{r}}_{i j} \text { and } \mathbf{F}_{i j}^{\mathrm{D}}=-\gamma_{i j} \omega^{\mathrm{D}}\left(r_{i j}\right)\left(\hat{\mathbf{r}}_{i j} \mathbf{v}_{i j}\right) \hat{\mathbf{r}}_{i j} \text {. }
$$

In Eq. (3), $\omega^{\mathrm{R}}\left(r_{i j}\right)=1-r_{i j} / r_{\mathrm{DPD}}, \omega^{\mathrm{D}}\left(r_{i j}\right)=\left[\omega^{\mathrm{R}}\left(r_{i j}\right)\right]^{2}$ are weight functions and vanish if $r_{i j}$ is greater than $r_{\mathrm{DPD}}$. Moreover, $\mathbf{v}_{i j}=\mathbf{v}_{i}-\mathbf{v}_{j}, \sigma^{2}=$ $2 \gamma k_{\mathrm{B}} T$, and the $\theta_{i j} \mathrm{~s}$ are randomly fluctuating variables with Gaussian statistics. The random and dissipative forces are coupled with the fluctuation-dissipation theorem and act as a thermostat [11], where the conventional parameterization is used for the dissipative force coefficient $\gamma$ as described in the work of Groot and Warren [14].

In this article we consider three sets of simulations: $i$ ) pure water, $i i$ ) pure methanol and iii) methanol/water mixtures. One molecule of water or methanol is represented by a coarse-grained bead. To define the self-repulsion parameter $a_{i i}$ of water beads, the conventional parameter obtained by fitting to the experimental isothermal compressibility of water, namely $25 k_{\mathrm{B}} T$, is used. However, in order to compute the pairwise interaction between the water and methanol beads $a_{i j}$, we make use of our recent extension of DPD parameterization applicable to variable bead volumes, since there is a significant difference between water and methanol pure liquid densities. We present the necessary formulas to compute the pairwise interactions in Eq. (4) but the full justification and derivation of the formula can be found in reference [21].

$$
\begin{aligned}
& \quad a_{i j}=\hat{a}_{i j}+\frac{p}{0.0454\left(a_{i i} \rho_{i, \text { pure }}+a_{j j} \rho_{j, \text { pure }}\right)} \chi_{i j} k_{\mathrm{B}} T, \hat{a}_{i j}=\sqrt{a_{i i} a_{j j}}, \\
& a_{i i}=\frac{p-\rho_{i, \text { pure }} k_{\mathrm{B}} T}{\alpha \rho_{i, \text { pure }}^{2} r_{\mathrm{DPD}}^{3}}
\end{aligned}
$$

In Eq. (4) $a_{i i}$ and $a_{j j}$ are the DPD interactions for the same type of beads, $\hat{a}_{i j}$ is the neutral interaction parameter which means there is no excess pressure generated upon mixing of $i$ and $j$ beads, and $\rho_{i \text {, pure is }}$ the dimensionless number density of the pure component $i$. The parameter $p$ represents the total system pressure applied to the DPD beads, for 
Table 1

Liquid state properties of beads used in simulations with mass density $\rho_{\mathrm{m}, i}$, molecular weight $M_{\mathrm{W}}$, number density $\rho_{i, \text { pure }}$, solubility parameter $\delta$, self-repulsive interaction $a_{i i}$, and interactions of bead $i$ with water $a_{i \text {-water }}$ in a 50:50 water/methanol mixture. $a_{\mathrm{i} \text {-water }}$ is the same as $a_{\mathrm{ii}}$ for water, therefore labelled as not applicable (N/A).

\begin{tabular}{lllllll}
\hline Type of bead & $\begin{array}{l}\rho_{\mathrm{m}, i} \\
{\left[\mathrm{~g} / \mathrm{cm}^{3}\right]}\end{array}$ & $\begin{array}{l}M_{\mathrm{W}} \\
{[\mathrm{g} / \mathrm{mol}]}\end{array}$ & $\begin{array}{l}\rho_{i, \text { pure }}=\rho_{m, i} / M_{\mathrm{W}} \\
{\left[\AA^{-3}\right]}\end{array}$ & $\delta$ & $\begin{array}{l}a_{i i} \\
{\left[\left(\mathrm{~J} / \mathrm{cm}^{3}\right)^{0.5}\right]}\end{array}$ & $\begin{array}{l}a_{i \text {-water }} \\
{\left[k_{\mathrm{B}} T\right]}\end{array}$ \\
\hline Methanol (M) & 0.787 & 32.04 & 0.0148 & 29.61 & 57.05 & 42.55 \\
Water $(\mathrm{W})$ & 1.000 & 18.02 & 0.0334 & 47.00 & 8.70 & $\mathrm{~N} / \mathrm{A}$ \\
\hline
\end{tabular}

which a value of $25.72 k_{\mathrm{B}} T / r_{\mathrm{DPD}}^{3}$ is obtained as computed from the DPD equation-of-state [14] with a set self-repulsion value of $25 k_{\mathrm{B}} T$, and a dimensionless number density of 3 . The coefficient $\alpha$ relates the excess pressure to the dimensionless number density in DPD. It usually takes the value of 0.101 above a number density value of 3 . However, for small number densities, we compute $\alpha$ from the fit reported in our earlier work [25]. Therefore, similar values of self-repulsion of $25 k_{\mathrm{B}} T$ are obtained from Eq. (4) for pure liquid methanol and pure water.

The degree of the mixing between water and methanol is characterized by the experimental Flory-Huggins interaction parameter $\chi_{i j}$ [39], obtained from the experimental solubility parameters $\delta$ by the following relation:

$\chi_{i j}=\frac{\bar{V}}{k_{\mathrm{B}} T}\left(\delta_{i}-\delta_{j}\right)^{2}$

Here $\bar{V}$ represents the average volume of a bead in the simulations computed from the average density by the relation,

$\bar{V}=\rho_{\text {mixture }}^{-1}=\left(\sum_{i} N_{i} \rho_{i, \text { pure }}^{-1}\right) / \sum_{i} N_{i}$

In Eq. (6), $N_{i}$ is the number of $i$ beads in the system. The densities of the pure components, solubility parameters, Flory-Huggins parameters and the DPD interaction parameters are adopted from our previous study [25]. The parameter $r_{\mathrm{DPD}}$ in Eq. (2) corresponds to the physical length scale of the DPD system. One can compute the actual value of $r_{\mathrm{DPD}}$ by using the relation $\rho_{\text {mixture }} r_{\mathrm{DPD}}^{3}=3$, where the right-hand-side of the equation is the dimensionless DPD bead number density in the simulation box. For example, a system composed of pure liquids of water and methanol have $r_{\text {DPD }}$ values of about $4.48 \AA$ and $5.87 \AA$, respectively. Moreover, for a water/methanol mixture with a 50:50 mixing ratio, $r_{\mathrm{DPD}}$ reads as $5.26 \AA$. As a result, the self-repulsion and pairwise repulsive parameters are computed as a function of $r_{\mathrm{DPD}}$ and takes different values as dictated by Eq. (4). In Table 1, in addition to the experimental density and solubility parameters, the $a_{i i}$ and $a_{i j}$ parameters are shown as computed for a 50:50 mixture of water and alcohol. The rest of the DPD simulation parameters of conservative interactions to study the pure liquids and different molar mixing ratios of water/ methanol mixtures are given in Table 2.
As dictated by Eq. (4), the self-repulsive interactions of the components are computed by setting a mechanical equilibrium in between pure components, thereby allowing these beads to represent their experimental pure-liquid densities in DPD simulations. As noted in Table 1, the strength of the self-repulsive interactions of methanol and water are different from each other. A smaller value of the selfrepulsive interaction of water means that the local density of water beads is much higher compared to that of methanol (i.e. methanol has higher self-repulsive interaction value). Although the self-repulsion strengths of the pure components are different from each other, the local-density difference leads to a similar force applied to each other following the DPD equation-of-state. However, the neutral interaction, which is the interaction between different bead types in case there is zero excess repulsion between them, is computed from the geometric average of the self-repulsive values (see Eq. (4)). The excess repulsion is eventually added by the contribution of the Flory-Huggins $\chi_{i j}$ parameter, therefore yielding the same repulsive strength as in GrootWarren's approach [14]. In line with these comments, the compressibilities of the individual systems represent the experimental compressibilities as noted later in the Results section.

The hydrogen bond interactions are essential in the structure creation of water and require careful attention [40]. In this work we used a similar approach as in our previous study, where the hydrogen bond interactions are defined as a separate potential term added to the conventional DPD non-bonded potential [25]. In that study the hydrogen bond interactions were only defined between the water and alcohol beads, and not between the pure species. This work is an extension of our previous efforts in modeling the hydrogen bonding interactions in DPD to the pure species as well. The Morse potential reads:

$V_{\text {Morse }}=D_{0}\left[\mathrm{e}^{-2 \sigma\left(r-r_{0}\right)}-2 \mathrm{e}^{-\sigma\left(r-r_{0}\right)}\right], r<r_{\text {DPD }}$

where $D_{0}$ is the depth of the potential well, $\sigma$ the width of the potential and $r_{0}$ the equilibrium distance of the hydrogen bonds. In our DPD simulations, the Morse potential is included if the pairwise distance is below a set cut-off distance $r_{\text {cut }}$.

Hydrogen bond interactions are directional; therefore, a certain angle characterizes the connection between the acceptor atom, the hydrogen atom and donor atom. In our parameterization, we do not consider an angular term in the Morse potential for several reasons. Firstly, there is no atomistic detail defined within a specific bead. This makes the definition of an angle impossible unless a certain virtual site, e.g. a multipole embedded in the bead or using pseudo-beads, is defined around a bead. We avoid to define such a virtual site since we consider computational efficiency as well. Secondly, a typical DPD time is reported to be about 90 ps by Groot and Rabone [41], which is obtained by matching the experimental self-diffusion coefficient of water to the one obtained via DPD simulations. It is known that the lifetime of the hydrogen bonds, in general, is about 1-20 ps and broken bond lifetimes are about 0.1 ps [42], which both are much smaller than the unit DPD

Table 2

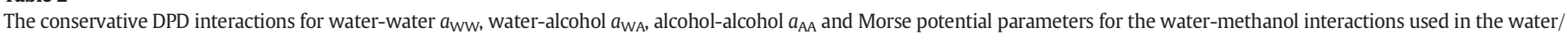

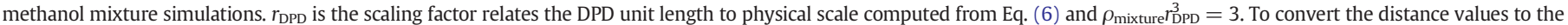
physical values, one has to scale with the corresponding $r_{\mathrm{DPD}}$ value.

\begin{tabular}{|c|c|c|c|c|c|c|c|c|}
\hline$x_{\text {Methanol }}$ & $x_{\text {Water }}$ & $\begin{array}{l}r_{\mathrm{DPD}} \\
{[\AA]}\end{array}$ & $\begin{array}{l}a_{\mathrm{WW}} \\
{\left[k_{\mathrm{B}} T\right]}\end{array}$ & $\begin{array}{l}a_{\mathrm{WA}} \\
{\left[k_{\mathrm{B}} T\right]}\end{array}$ & $\begin{array}{l}a_{\mathrm{AA}} \\
{\left[k_{\mathrm{B}} T\right]}\end{array}$ & $\begin{array}{l}D_{0} \\
{\left[k_{\mathrm{B}} T\right]}\end{array}$ & $r_{0}$ & $r_{\text {cut }}$ \\
\hline 0.000 & 1.000 & 4.48 & 25.0 & $\mathrm{~N} / \mathrm{A}$ & $\mathrm{N} / \mathrm{A}$ & 12.0 & 0.62 & 0.72 \\
\hline 0.125 & 0.875 & 4.70 & 18.3 & 57.5 & 126.8 & 11.4 & 0.61 & 0.75 \\
\hline 0.250 & 0.750 & 4.91 & 13.8 & 48.4 & 93.6 & 13.2 & 0.59 & 0.72 \\
\hline 0.375 & 0.625 & 5.09 & 10.9 & 44.1 & 72.8 & 16.1 & 0.57 & 0.69 \\
\hline 0.500 & 0.500 & 5.27 & 8.7 & 42.6 & 57.8 & 18.3 & 0.55 & 0.67 \\
\hline 0.625 & 0.375 & 5.43 & 7.0 & 43.1 & 46.3 & 21.8 & 0.53 & 0.65 \\
\hline 0.750 & 0.250 & 5.59 & 5.8 & 45.5 & 37.8 & 23.5 & 0.52 & 0.63 \\
\hline 0.875 & 0.125 & 5.74 & 4.8 & 49.2 & 31.4 & 27.0 & 0.50 & 0.62 \\
\hline 1.000 & 0.000 & 5.87 & N/A & N/A & 25.0 & 11.0 & 0.52 & 0.65 \\
\hline
\end{tabular}


time. Thence, in our representation, the Morse potential lacks the directionality constraints and represents the overall hydrogen bond interactions. The rest of the non-bonded potential is the conventional purely repulsive DPD potential.

Generally, the characterization of hydrogen bonds from atomistic simulations requires setting specific angle and distance values. Within this set of criteria, a hydrogen bond is defined to be present or not, therefore making the existence of a hydrogen bond dependent on the set criteria. Our parameterization scheme takes the Morse parameters directly from experimental values removing such dependencies, as reported and discussed in the Results and Discussion section.

\subsection{Simulation details}

The DPD simulations in this work are run with the LAMMPS simulator [43]. NVT conditions are employed in all simulations except that NPT conditions are used to study the isothermal compressibility of water and methanol, and the excess volumes of water/methanol mixtures. The initial simulation box dimensions are $20 \times 20 \times 20 r_{\text {DPD }}^{3}$ and contains 24,000 beads corresponding to an average number density of 3 . A pressure value of $25.72 k_{\mathrm{B}} T / r_{\mathrm{DPD}}^{3}$ is set in the NPT simulations.

A series of simulation boxes are constructed for $i$ ) pure water $i i$ ) pure methanol and iii) water/methanol mixtures with methanol molar fractions of $0.125,0.250,0.375,0.50,0.625,0.750,0.875$. The total duration of each simulation is $1 \times 10^{6}$ time-steps with the first $5 \times 10^{5}$ time-steps for equilibration and the rest for data collection. The time step in the simulations is selected as $\Delta t=0.001 t_{\mathrm{DPD}}$. Periodic boundary conditions are employed in all dimensions. Hydrogen bonding interactions are switched-on at all times in the simulations.

\section{Results and discussion}

\subsection{Morse potential parameters for the simulation of water}

The most crucial step in simulating the liquid water is to define the non-bonded interaction parameters at the DPD scale. As mentioned, the non-bonded force acting on a particular bead is composed of the conservative potential of the DPD interactions and the added Morse potential representing the hydrogen bond interactions. The conventional DPD interaction with a strength of $25 k_{\mathrm{B}} T$ is adopted for the conservative potential part. For the hydrogen bond interactions, the Morse potential is used in which we require to parameterize three parameters as noted in Eq. (7): $i$ ) The hydrogen bond strength $D_{0}$, ii) the equilibrium hydrogen bond length $r_{0}$ and iii) the force constant representing the width of the potential $\sigma$.

The parameters of the Morse potential are defined by taking experimental data as reference. Although there is no standard definition for the hydrogen bond energy value, the strength of the attraction between water molecules in liquid water corresponds to about $23.3 \mathrm{~kJ} / \mathrm{mol}(\sim 9.4$ $k_{\mathrm{B}} T$ ) [44]. This energy is actually the energy required to break the bond, which is about half of the energy of vaporization at $25^{\circ} \mathrm{C}$. Moreover, during the formation and dissociation of the hydrogen bonds in the liquid state, the rearrangements of the molecules require an additional energy of about $6.3 \mathrm{~kJ} / \mathrm{mol}\left(\sim 2.5 k_{\mathrm{B}} T\right)$ [45]. In our simulations the Morse potential constitutes the effective non-bonded attractive part of the interactions between water beads. In addition, within a typical DPD time step, hydrogen bonds between water beads form and break since the DPD time step is larger than the typical time required for the formation and dissociation of the hydrogen bonds. Therefore, these two energy values add together in a typical DPD time. Hence, in our simulations we take a $D_{0}$ value of $29.6 \mathrm{~kJ} / \mathrm{mol}\left(\sim 12 k_{\mathrm{B}} T\right)$ representing the total attraction of the hydrogen bonds.

In our simulations, the hydrogen bonds form (and break) if their pairwise separation falls within (outside) a certain distance value $r_{\text {cut }}$. For the cut-off value of the formation of the hydrogen bonds different values are reported. Some studies report a value of about $3.10 \AA$ [46], on the other hand, others use a higher value of about $3.50 \AA$ [47]. In our simulations, a cut-off value $r_{\text {cut }}$ of $3.22 \AA$ ( $\left.0.72 r_{\text {DPD }}\right)$ is used to represent the pairwise interaction length where the hydrogen bonds form. The parameter $r_{0}$ for the equilibrium hydrogen bond distance is directly taken as the experimental distance of the $0 \cdots 0$ interaction in a four-coordinated hydrogen-bonded liquid water, which reads as $2.80 \AA\left(\sim 0.62 r_{\mathrm{DPD}}\right)$. The remaining parameter $\sigma$, defining the width of the Morse potential, is taken as 6, representing a narrow width. The value is selected consistent with previous studies $[19,48]$ and observed to represent the structure of liquid water.

\subsection{Simulations of liquid water}

The Morse potential parameters are initially tested to monitor the structure of liquid water in the DPD simulations. To that purpose, we compute the RDF profiles and compare the results with both the DPD simulation that does not consider hydrogen bond attraction and an atomistic reference [7].

It is clear from Fig. 1 that the DPD simulations with the hydrogen bond extension represents the characteristic liquid-state water RDF reasonably well. Although both the locations of the first neighboring shell and the first minima are predicted nicely as compared to the atomistic simulation, the corresponding (maximum and minimum) values are slightly underestimated by the DPD simulation. However, the secondary and the tertiary neighbor characteristics are predicted in very good agreement. As expected, the RDF of the classical DPD simulations, where there are no hydrogen bond interactions present, are far off from the atomistic one.

Water is known to form a structure with a coordination number of about 4 as a result of the tetrahedral structure. In ice this tetrahedral configuration, arising from the presence of two donors and two acceptors per molecule, is fairly complete. In liquid water the tetrahedral configuration can also be found, though less frequently, because the (somewhat larger) thermal energy leads to a number of fivecoordinated configurations. To assess the coordination numbers $(\mathrm{CN})$, we compute the CNs from the RDF profiles using the conventional relation,

$C N=4 \pi \rho \int_{0}^{r_{1}} r^{2} g(r) \mathrm{d} r$

where $r_{1}$ indicates the size of the first coordination shell as read from the first minimum $r_{1}$ and $\rho$ is the number density. The computed $\mathrm{CN}$

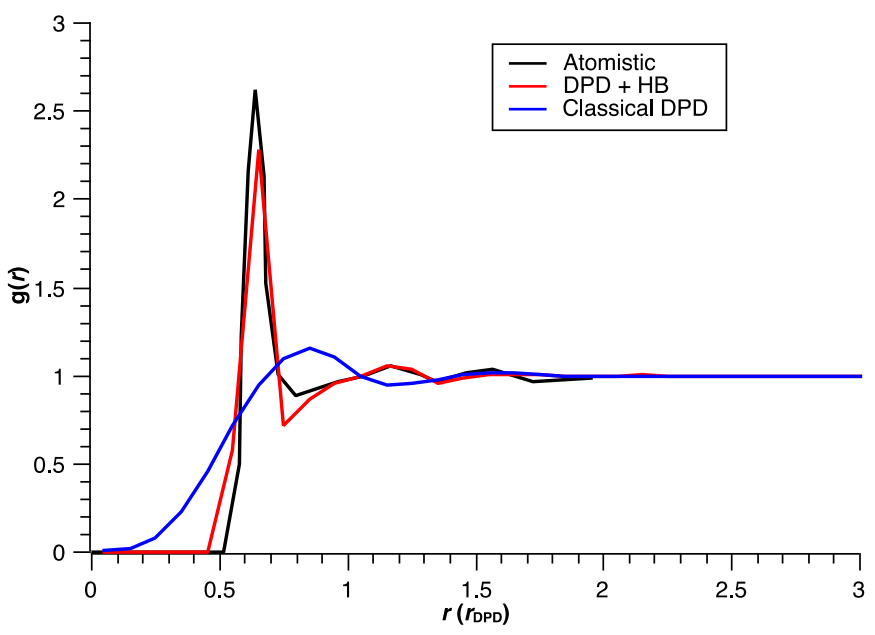

Fig. 1. Radial Distribution Functions $g(r)$ of the DPD simulations of pure water with the hydrogen bond interactions (red), classical DPD simulations that do not contain hydrogen bond attraction (blue) and the atomistic RDF (black) [7]. The distance values of the atomistic RDF are converted to the DPD units with the proper length scale conversion, which is $4.48 \AA$ 
values are: 4.95 (atomistic simulations), 4.86 (DPD simulations with the hydrogen bond interactions), 3.86 (classical DPD simulations). As will be clear, the $\mathrm{CN}$ values for the atomistic and DPD simulations including hydrogen bond extension are in a quite good agreement, although both are somewhat larger than the experimental value 4.4 [49]. However, the classical DPD simulations significantly underestimate these predictions. The fact that liquid water contains to some extent five-coordinated water is represented in our simulations.

The three-body angular distribution is another property that defines the structure of liquid water. This distribution, together with the threebody distance-dependent term, actually forms the three-body distribution contribution to the many-body potential. In other words, the many-body potential that is acting on a particular bead depends on the three-body angular distribution, as can be seen in the Stillinger-Weber potential $[7,50]$. Therefore, a proper representation of the three-body angular distribution is needed for the correct potential of mean force (PMF). For the case of liquid water, the three-body angular distribution is a bimodal distribution, which is an indication of the tetrahedral configuration. In Fig. 2, the three-body angular distributions obtained with the DPD simulations including hydrogen bond attraction are plotted and compared with the classical DPD simulations as well as with the literature data.

In Fig. 2 the two MD results taken from literature $[7,8]$ are obtained in approximate agreement by employing SPC and SPC/E force fields, respectively, as can be seen from the difference in width for the first peak and the different position of the minimum. Our DPD results show a slightly broader first peak, followed by a somewhat lower height for the second peak as compared to the reference profiles. The values of these two major peaks at $55^{\circ}$ and $110^{\circ}$ are observed to be slightly off from the atomistic references, which are $50^{\circ}$ and $100^{\circ}$, respectively. It is clear from Fig. 2 that the DPD simulations including the hydrogen bond attraction reproduce the tetrahedral structure reasonably well. The slight deviation from the atomistic profiles can be attributed to the five-coordinated water beads as also noted by the coordination numbers. Nevertheless, the three-body angular distribution is represented to a great extent by our DPD simulations. Needless to say, that the three-body angular distribution of the classical DPD simulation fails completely in reproducing the bimodal distribution of the threebody angle values.

Finally, we performed a computation of the isothermal compressibility to demonstrate that the hydrogen bonded DPD system has the proper experimental value. To that purpose we performed NPT simulations with different number densities $\rho$ and computed the isothermal

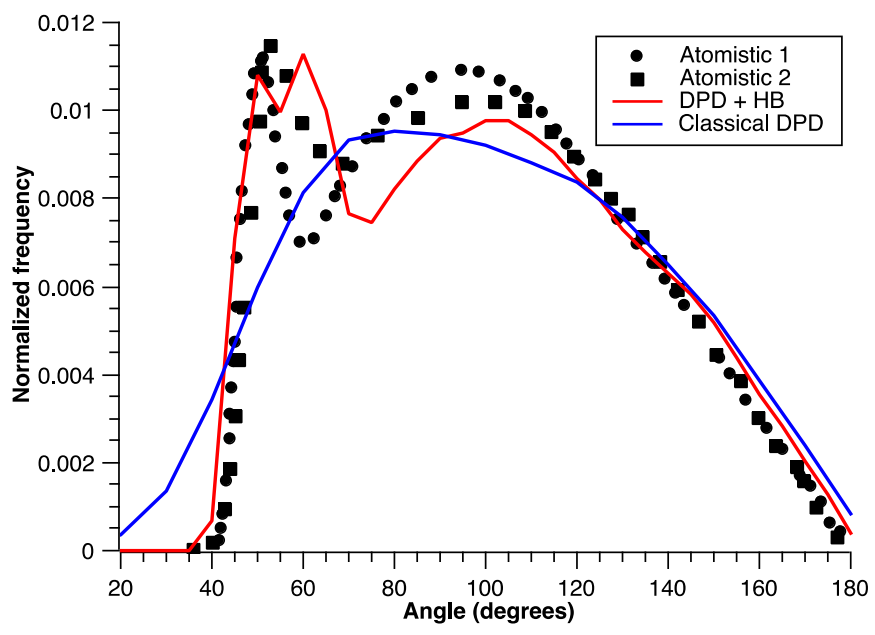

Fig. 2. Three-body angular distributions obtained by the DPD simulations with the hydrogen bond attraction (red) and classical DPD simulations (blue) compared with two references: Atomistic 1 [8] and Atomistic 2 [7]. The small dip in the first peak of the $\mathrm{DPD}+\mathrm{HB}$ simulation result is considered to be due to statistical fluctuation. compressibility using

$\kappa_{T}=\frac{1}{k_{\mathrm{B}} T} \frac{V^{2}-V^{2}}{V}$ and $\kappa^{-1}=\frac{1}{\rho k_{\mathrm{B}} T \kappa_{T}}$

where $\kappa^{-1}$ and $\kappa_{T}$ indicate the dimensionless and the experimental isothermal compressibilities, respectively. In Eq. (9), $\langle\ldots\rangle$ stands for the ensemble averages, $V$ for the simulation box volume and $\rho$ for the number density of molecules. The dimensionless isothermal compressibility has the experimental value of about 16.1 for pure water at room temperature [14,51]. According to Eq. (9), the computed isothermal compressibility of the DPD simulations including the hydrogen bond attraction is 20.8. As seen from the RDFs in Fig. 1, the Morse potential leads to a harder structure than the classical DPD estimation confirming the obtained higher compressibility. Nevertheless, a somewhat higher but comparable isothermal compressibility value is obtained as compared with the experimental value for the DPD simulations with the hydrogen bond addition.

\subsection{Simulations of pure liquid methanol}

Liquid methanol demonstrates a strong hydrogen bonded network [52]. There is a hydrogen bond attraction between the oxygen and hydrogen atoms of the hydroxyl groups of different methanol molecules. In this section, we study the role of the hydrogen bond addition into DPD to predict the structure and physical properties of pure methanol in order to test whether the parameterization procedure is applicable to other pure and binary systems.

To that purpose, the self-repulsive methanol interaction used is taken $25 k_{\mathrm{B}} T$, as dictated by its pure liquid density and a DPD dimensionless density of 3 as discussed earlier. Moreover, the hydrogen bond attraction between pure methanol beads is considered as well to be similar to that of pure water beads as presented in this work and thus we use a similar pair potential energy of about $21 \mathrm{~kJ} / \mathrm{mol}$ [53] ( 8.5 $k_{\mathrm{B}} T$ ) with an extra energy required for the rearrangement of the methanol molecules of $6.3 \mathrm{~kJ} / \mathrm{mol}\left(\sim 2.5 k_{\mathrm{B}} T\right)$, which makes the total interaction energy $27.3 \mathrm{~kJ} / \mathrm{mol}\left(\sim 11 k_{\mathrm{B}} T\right)$. The pairwise distance and a cut-off value $r_{\text {cut }}$ of the hydrogen bond interactions of methanol molecules is reported in literature as about $3.2 \AA$ and $3.8 \AA$, respectively [53]. In our simulations, the value used to represent the equilibrium hydrogen bond distance is $0.52 r_{\text {DPD }}(\sim 3.1 \AA)$ and the cut-off value $r_{\text {cut }}$ to define a formed hydrogen bond is taken as $0.65 r_{\text {DPD }}(\sim 3.8 \AA)$. These values deviate slightly from the reported values, but are observed to represent the structure better than the literature values. The width of the Morse potential is kept as 6 as indicated before.

In Fig. 3 the first peaks of both the atomistic and DPD simulations are quite similar to each other. However, the second and third peaks are slightly lower as well as shifted somewhat as compared to the MD simulations [54] employed by using a polarizable form of the TraPPE potential for methanol, signifying a longer-range order for the second and third shell interactions in the MD simulations. The set Morse potential parameters yield a good estimate of the first shell interactions, which are more concentrated than the remaining neighboring shells. Although, there is a difference for the second and third peaks a much better estimate of the structure is obtained when the hydrogen bond interactions are incorporated in the DPD simulations as compared to the classical DPD.

The experimental isothermal compressibility of pure liquid methanol at room temperature is about $1.27 \times 10^{-9} \mathrm{~Pa}^{-1}$ [55], yielding a dimensionless isothermal compressibility value of about 13.7. Upon employing constant pressure NPT simulations to pure methanol, we estimate a dimensionless isothermal compressibility value of 18.3. Similar to pure water, this value is on the high side as a result of the harder structure obtained compared to the classical DPD simulations. Nevertheless, we notice that the decreasing trend of the predicted isothermal 


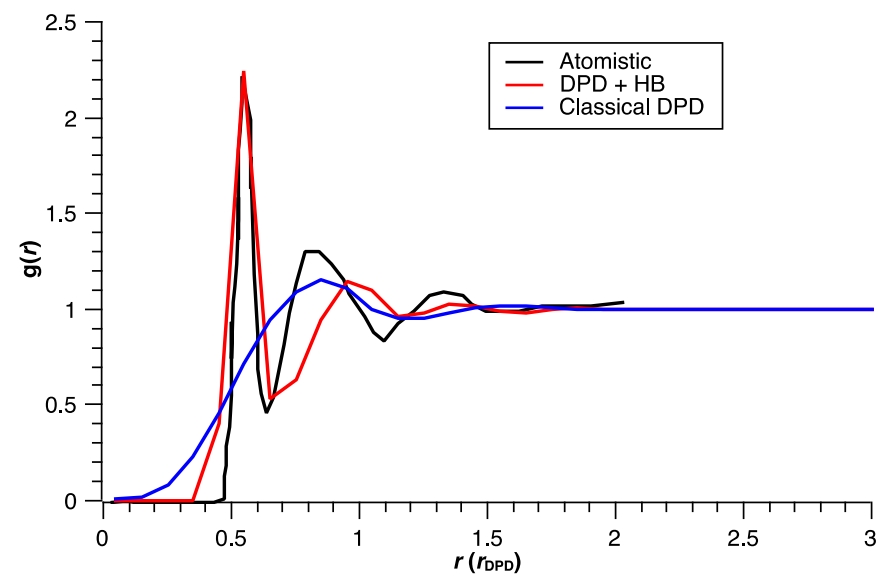

Fig. 3. Radial Distribution Functions $g(r)$ of the DPD simulations of pure methanol with the hydrogen bond interactions (red), classical DPD simulations that do not contain hydrogen bond attraction (blue), and the atomistic RDF (black) [54].

compressibility value of methanol is similar to the decreasing trend in the experimental values when compared with water.

\subsection{Simulations of methanol/water mixtures}

The hydrogen bond addition leads to an estimation of the proper structure with a reasonable agreement for the pure liquids. In this section, we perform DPD simulations to test the applicability of the hydrogen bond added DPD simulations in predicting the properties of the binary mixtures of water and methanol. As mentioned before, the conservative DPD parameters of both self-repulsion and the pairwise interactions are dictated by the molar fractions of the compounds in the mixture (see Eq. (6) and Table 2) due to the change of the physical length scale $r_{\mathrm{DPD}}$ computed as a function of the average density of the binary mixture. Therefore, the Morse potential parameters, namely the equilibrium hydrogen bond distance and the hydrogen bond cut-off values, need to be rescaled with respect to the change in $r_{\mathrm{DPD}}$.

In water/methanol mixture simulations, the Morse potential interactions are set between water-water, water-methanol and water-water beads. For water-water and methanol-methanol, the same distance and energy values in the Morse potential are used as reported earlier with the proper scaling of the distances for each mixture. For example, a $r_{\text {DPD }}$ value of $5.26 \AA$ is used for the length scale conversion for a 50:50 M mixture of water and methanol. For the water-methanol Morse potential interactions we employ an arithmetic average mixing rules for the distances. In other words, arithmetic averages of the pure water and pure alcohol parameters are taken to compute the equilibrium and the cut-off distances of the hydrogen bonds. The width of the Morse potential (the force constant) is kept the same as for the pure components, namely 6 , but the hydrogen bond energy parameters in the Morse potential $D_{0}$ of the water-methanol interactions are estimated by a fitting of the experimental negative excess volumes. The hydrogen bond energy values that represent the experimental negative excess volumes best with the set equilibrium hydrogen bond distance $r_{0}$ and cut-off values $r_{\text {cut }}$ are presented in Table 2 together with all simulation parameters that are used in water/methanol mixture simulations, i.e. the conservative DPD interactions and the Morse potential parameters of water-water, water-methanol and methanol-methanol.

In Table 2, the difference between the self-repulsive and pairwise interactions can be attributed to the change in the physical length scale of the DPD unit length $r_{\mathrm{DPD}}$. Although the value changes with respect to the change in molar fraction of the components in the mixture, the pressure generated by each pure component is the same as read from the DPD equation-of-state. The excess repulsion in between water and alcohol is computed from the experimental Flory-Huggins parameters as discussed before.
In Fig. 4 the experimental negative volume excess data [56] are plotted as the total volume change by a division of the excess volume $V^{E}$ to the ideal volume $V^{\text {ideal }}$ as read from

$V^{\mathrm{E}}=\frac{x_{\mathrm{A}} M_{\mathrm{A}}+x_{\mathrm{W}} M_{\mathrm{W}}}{\rho_{\mathrm{L}}}-x_{\mathrm{A}} V_{\mathrm{m}, \mathrm{A}}-x_{\mathrm{W}} V_{\mathrm{m}, \mathrm{W}}$.

In Eq. (10) $x_{i}$ represents the molar fractions of liquid $i, \rho_{\mathrm{L}}$ the liquid density of the mixture, $V_{i}$ the molar volume, and $M_{i}$ the molecular weight of each component $i$. The term $i$ represents both the alcohol A and water $\mathrm{W}$ beads. The first term is the mixture volume and the last two terms represent the volume of the ideal mixture.

In the DPD simulations, the excess volume $V^{\mathrm{E}}$ is the difference between the simulated volume including all the hydrogen bond interactions and the ideal volume. The ideal volume values in the DPD simulations are obtained by switching off the water-methanol hydrogen bond interactions, but keeping the intra-molecular water-water and methanol-methanol hydrogen bond interactions. As mentioned, the $D_{0}$ parameters in Table 2 are obtained by fitting the experimental volumetric data in Fig. 4. As can be seen in the figure, a good estimate of the experimental data is reached by the set $D_{0}$ values and the computed $r_{0}$ and $r_{\text {cut }}$ values. The hydrogen bond energy values can be represented by a linear function of the methanol molar fraction given by $D_{0}$ $(x)=20.89 x_{\text {methanol }}+8.31$. This relation is somewhat different than used in our earlier work (see Supplementary Information of reference [25]) which was $D_{0}(x)=12.37 x_{\text {methanol }}+3.81$. The difference and higher values of $D_{0}$ in the current study can be attributed to the extra attraction between water-water and methanol-methanol beads due to the inclusion of hydrogen bond interactions, which were absent in our earlier work. The presence of the hydrogen bonds as intra-molecular interactions of water and methanol eventually require a higher $D_{0}$ value between water and methanol to yield a similar volume excess behavior. The classical DPD simulations without the hydrogen bond contribution always lead to a positive excess value, as noted in our previous work [25].

In this part, we compute the RDFs to assess the structure of the methanol-methanol, methanol-water and water-water pairs in a 50:50 water/methanol mixture. The literature data that are obtained using atomistic simulations [7] obtained by using OPLS-AA force field for methanol and SPC model for water are compared with our estimates and plotted in Fig. 5.

In Fig. 5, as a result of the DPD simulations including the hydrogen bond attraction, the RDFs of the same and different bead pairs are

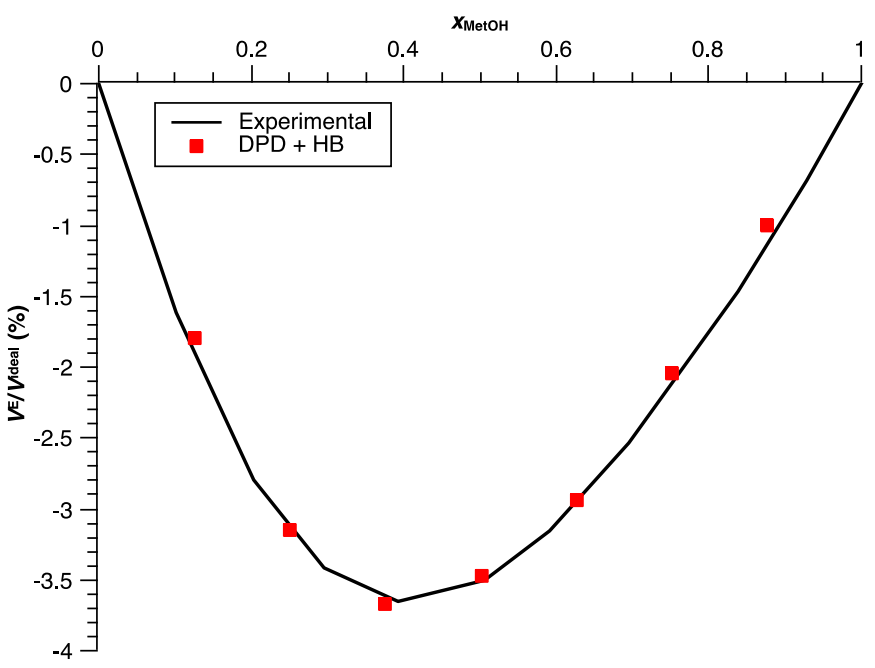

Fig. 4. Percent total volume change $\left(V^{\mathrm{E}} / V^{\text {ideal }}\right)$ change as a function of methanol molar fraction in water mixture. Solid black curve is the experimental data [56] and the red squares are the DPD simulations with the hydrogen bond attraction. 
reproduced in a good qualitative but only in fair quantitative agreement with the atomistic simulations. The qualitative agreement is present since the peak locations, the number of the peaks and the width of the
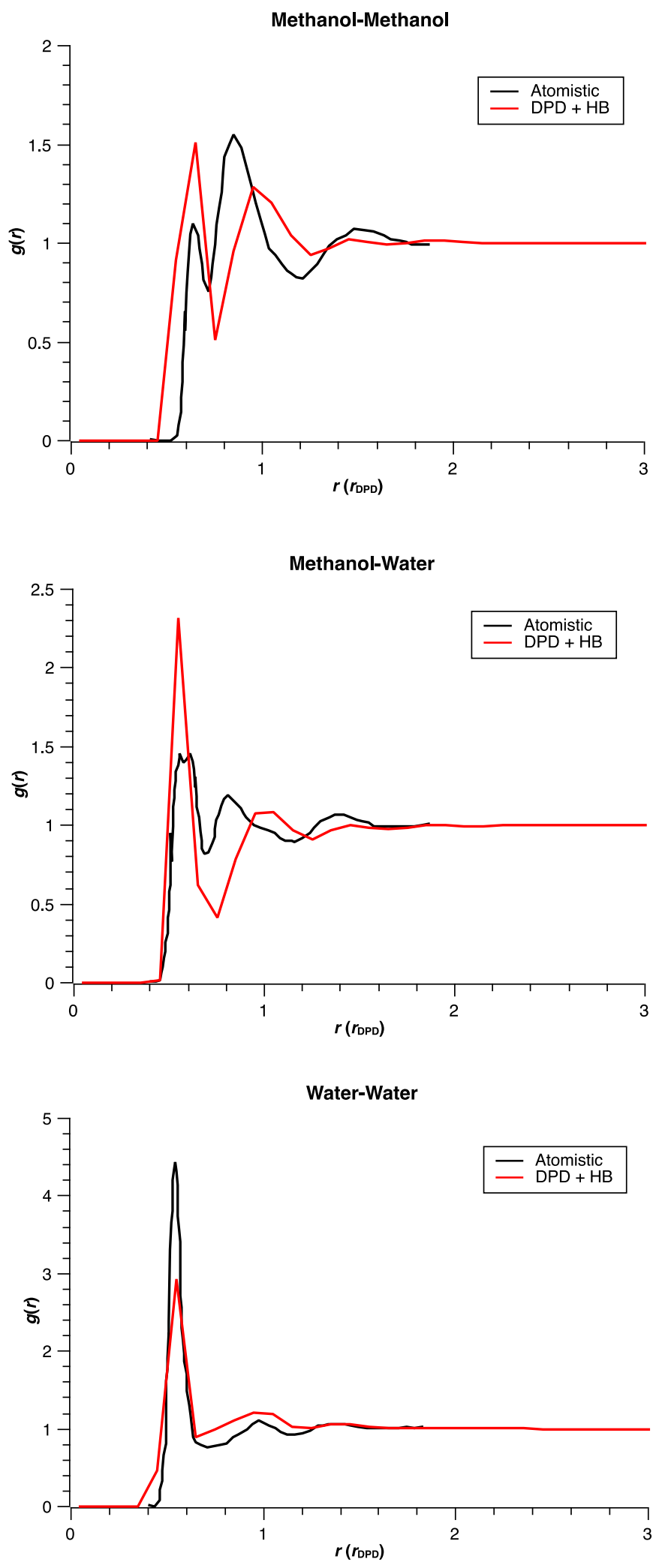

Fig. 5. Radial Distribution Functions plotted for methanol-methanol, methanol-water and water-water molecules in a 50:50 water/methanol molar mixture. The atomistic data are adapted from the reference [7]. The physical distance values of the literature RDFs are scaled to the DPD length scale with a scaling factor of $5.26 \AA$. peaks are predicted successfully. On the other hand, the $g(r)$ values are somewhat off from our prediction for all three RDF combinations. Nevertheless, we assess the intrinsic nature of the structure of the similar and different pairs in a water/methanol mixture as in reasonable agreement with the atomistic data.

There are some phase shifts in the second peaks of the methanolmethanol and methanol-water RDF profiles. A possible reason is that in the reference work of Voth et al. the coarse-graining is done based on the center-of-mass coordinates for methanol and center-ofgeometry coordinates for water molecules. To give a feeling, there is about a $0.2 \AA$ Aifference between the center-of-mass and center-ofgeometry of methanol molecules. In our DPD simulations, we do not employ a specific coarse-graining scheme to define the coordinates of the beads. A coarse-grained bead in DPD is identified based on the type of the molecule and is not based on its geometry, for which the exact location of the center of a particular bead is unknown. For homogenous systems (i.e. pure systems and systems with similar sizes), this difference would be of minor importance, since the definition of the exact location of the center of a bead is the same for all the beads. However, methanol and water have a significant difference in their pureliquid densities, therefore sizes, which makes defining the exact location of the bead geometry more important.

\section{Conclusions}

In this work we report a modeling procedure and parameters that can be used within DPD to mimic the structure and properties of water and methanol in their pure liquid states as well as in their binary mixtures. The modeling procedure is adopted from our previous work, where the hydrogen bond interactions are defined as an added Morse potential to the conservative DPD potential. The sensitive balance between Morse potential parameters and the non-bonded DPD potential requires a careful attention in their parameterization. The experimental evaporation enthalpy is used to represent the Morse bond strength parameters for pure water and pure methanol. Moreover, experimental literature data is taken as a reference to set the equilibrium hydrogen bond and the cut-off distances to define the hydrogen bond interactions. For pure water the RDF, the coordination number, the isothermal compressibility and the three-body angular distributions for the tetrahedral structure are predicted in a good extent consistent with the experiments. In pure methanol, the structure and the isothermal compressibility predictions are slightly off but predicting the characteristic behavior of the atomistic structure. To test the applicability of the proposed procedure in simulating liquid mixtures, we simulated methanol/water mixtures to initially identify the simulation parameter $D_{0}$. The simulations resulted in a good correspondence of the excess volumes with the experimentally observed negative excess volumes and yield results that are in general represents the intrinsic characteristics of the RDF profiles for the individual and pairs in the mixture. To conclude, initially, we were motivated and started the present paper to test the proposed procedure for pure liquid water, but our findings can be seen as a simple attempt to correctly simulate the structure of liquid mixtures where hydrogen bonds play a crucial role in DPD calculations. Clearly, although the results for the pure liquids and their mixtures represent the experimental behavior fairly well, further refinement still can be made. Both the temperature and energy are ill-defined in DPD. Therefore, other properties like heat capacity and thermal expansion coefficient are rather better quantified by employing atomistic molecular dynamics simulations performed with the coordinates that are reverse-mapped from DPD coordinates. We expect that a two-bead representation for methanol, which is planned for a future study, produces better agreement with experimental RDF. This probably applies as well to the RDF's for the mixtures. Finally, the present approach deals mainly with the static properties and the dynamic properties, such as hydrogen bond lifetime and diffusivity, are left for future work. 


\section{CRediT authorship contribution statement}

Gokhan Kacar:Conceptualization, Methodology, Software, Validation, Formal analysis, Investigation, Writing - original draft, Visualization.Gijsbertus de With:Conceptualization, Methodology, Writing - review \& editing.

\section{Declaration of competing interest}

The authors declare that they have no known competing financial interests or personal relationships that could have appeared to influence the work reported in this paper.

\section{Acknowledgments}

Part of the work is financially supported by the TUBITAK 2232 Reintegration Grant (no. 116C003).

\section{References}

[1] A. Laio, M. Parrinello, Proc. Natl. Acad. Sci. U. S. A. 99 (2002), 12562.

[2] D. Reith, M. Putz, F. Muller-Plathe, J. Comput. Chem. 24 (2003) 1624.

[3] A.P. Lyubartsev, A. Laaksonen, Phys. Rev. E 52 (1995) 3730.

[4] S. Izvekov, G.A. Voth, J. Chem. Phys. 123 (2005).

[5] S. Izvekov, G.A. Voth, J. Phys. Chem. B 109 (2005) 2469.

[6] W.G. Noid, J.W. Chu, G.S. Ayton, V. Krishna, S. Izvekov, G.A. Voth, A. Das, H.C. Andersen, J. Chem. Phys. 128 (2008).

[7] L.Y. Lu, J.F. Dama, G.A. Voth, J. Chem. Phys. 139 (2013).

[8] C. Scherer, D. Andrienko, Phys. Chem. Chem. Phys. 20 (2018), 22387.

[9] V. Ruhle, C. Junghans, A. Lukyanov, K. Kremer, D. Andrienko, J. Chem. Theory Comput. 5 (2009) 3211.

[10] P.J. Hoogerbrugge, J.M.V.A. Koelman, Europhys. Lett. 19 (1992) 155.

[11] P. Español, P. Warren, Europhys. Lett. 30 (1995) 191.

[12] U. Frisch, B. Hasslacher, Y. Pomeau, Phys. Rev. Lett. 56 (1986) 1505.

[13] A.J.C. Ladd, J. Fluid Mech. 271 (1994) 285.

[14] R.D. Groot, P.B. Warren, J. Chem. Phys. 107 (1997) 4423.

[15] P. Español, P.B. Warren, J. Chem. Phys. 146 (2017).

[16] A.C.C. Esteves, K. Lyakhova, L.G.J. van der Ven, R.A.T.M. van Benthem, G. de With, Macromolecules 46 (2013) 1993.

[17] G. Kacar, E.A.J.F. Peters, L.G.J. van der Ven, G. de With, Phys. Chem. Chem. Phys. 17 (2015) 8935.
[18] C.H. Li, X.W. Fu, W.H. Zhong, J. Liu, Acs Omega 4 (2019) 10216

[19] A. Vishnyakov, D.S. Talaga, A.V. Neimark, J. Phys. Chem. Lett. 3 (2012) 3081.

[20] G. Kacar, E.A.J.F. Peters, G. de With, Soft Matter 9 (2013) 5785.

[21] G. Kacar, E.A.J.F. Peters, G. de With, Europhys. Lett. 102 (2013).

[22] M.T. Lee, A. Vishnyakov, A.V. Neimark, J. Chem. Theory Comput. 11 (2015) 4395

[23] A. Vishnyakov, R. Mao, M.T. Lee, A.V. Neimark, J. Chem. Phys. 148 (2018).

[24] K.P. Santo, A. Vishnyakov, R. Kumar, A.V. Neimark, Macromolecules 51 (2018) 4987

[25] G. Kacar, G. de With, Phys. Chem. Chem. Phys. 18 (2016) 9554

[26] G. Kacar, Chem. Phys. Lett. 690 (2017) 133.

[27] G. Kacar, Phys. Chem. Chem. Phys. 20 (2018), 12303.

[28] G. Kacar, Colloid Polym. Sci. 297 (2019) 1037-1051.

[29] J. Zielkiewicz, J. Chem. Phys. 123 (2005).

[30] L. Darre, M.R. Machado, S. Pantano, WIREs Comput. Mol. Sci. 2 (2012) 921.

[31] K.R. Hadley, C. McCabe, Mol. Simul. 38 (2012) 671.

[32] T. Urbic, Mol. Simul. 45 (2019) 279.

[33] T. Urbic, K.A. Dill, J. Am. Chem. Soc. 140 (2018), 17106.

[34] J. Michalowsky, L.V. Schafer, C. Holm, J. Smiatek, J. Chem. Phys. 146 (2017).

[35] S.O. Yesylevskyy, L.V. Schafer, D. Sengupta, S.J. Marrink, PLoS Comput. Biol. 6 (2010).

[36] M.S. Deng, H.J. Shen, J. Phys. Chem. B 120 (2016) 733.

[37] E.K. Peter, I.V. Pivkin, J. Chem. Phys. 141 (2014).

[38] R. Vaiwala, S. Jadhav, R. Thaokar, Mol. Simul. 44 (2018) 540.

[39] P.J. Flory, Principles of Polymer Chemistry, Cornell University Press, Ithaca, New York, 1953.

[40] F.H. Stillinger, Theory and Molecular Models for Water, 1 edn John Wiley \& Sons, Inc, New York, 1975.

[41] R.D. Groot, K.L. Rabone, Biophys. J. 81 (2001) 725.

[42] F.N. Keutsch, R.J. Saykally, Proc. Natl. Acad. Sci. U. S. A. 98 (2001), 10533.

[43] S. Plimpton, J. Comput. Phys. 117 (1995) 1.

[44] S.J. Suresh, V.M. Naik, J. Chem. Phys. 113 (2000) 9727.

[45] J.D. Smith, C.D. Cappa, K.R. Wilson, B.M. Messer, R.C. Cohen, R.J. Saykally, Science 306 (2004) 851.

[46] A. Khan, J. Phys. Chem. B 104 (2000), 11268.

[47] M.T.C.M. Costa, J. Mol. Struct. (THEOCHEM) 729 (2005) 47.

[48] S.W. Chiu, H.L. Scott, E. Jakobsson, J. Chem. Theory Comput. 6 (2010) 851.

[49] A.H. Narten, H.A. Levy, in: F. Franks (Ed.), The Physics and Physical Chemistry of Water, Springer New York, Boston, MA 1972, pp. 311-332.

[50] F.H. Stillinger, T.A. Weber, Phys. Rev. B 31 (1985) 5262.

[51] W. Brostow, T. Grindley, M.A. Macip, Mater. Chem. Phys. 12 (1985) 37.

[52] J.A.B. da Silva, F.G.B. Moreira, V.M.L. dos Santos, R.L. Longo, Phys. Chem. Chem. Phys. 13 (2011) 593.

[53] M. Matsumoto, K.E. Gubbins, J. Chem. Phys. 93 (1990) 1981.

[54] P.G. Lafond, S. Izvekov, J. Chem. Theory Comput. 12 (2016) 5737.

[55] M. Taravillo, F.J. Perez, J. Nunez, M. Caceres, V.G. Baonza, J. Chem. Eng. Data 52 (2007) 481.

[56] A. Arce, A. Blanco, A. Soto, I. Vidal, J. Chem. Eng. Data 38 (1993) 336. 